\title{
Three-Dimensional Paper-Based Microfluidic Device for Assays of Protein and Glucose in Urine
}

\author{
Deidre Sechi, Brady Greer, Jesse Johnson, and Nastaran Hashemi* \\ Department of Mechanical Engineering, Iowa State University, Ames, Iowa, United States
}

\begin{abstract}
The first step in curing a disease is being able to detect the disease effectively. Paper-based microfluidic devices are biodegradable and can make diagnosing diseases costeffective and easy in almost all environments. We created a three-dimesnional (3D) paper device using wax printing fabrication technique and basic principles of origami. This design allows for a versatile fabrication technique over previously reported patterning of SU-8 photoresist on chromatography paper by employing a readily available wax printer. The design also utilizes multiple colorimetric assays that can accommodate one or more analytes including urine, blood, and saliva. In this case to demonstrate the functionality of the 3D paper-based microfluidic system, a urinalysis of protein and glucose assays is conducted. The amounts of glucose and protein introduced to the device are found to be proportional to the color change of each assay. This color change was quantified by use of Adobe Photoshop. Urine samples from participants with no pre-existing health conditions and one person with diabetes were collected and compared against synthetic urine samples with predetermined glucose and protein levels. Utilizing this method, we were able to confirm that both protein and glucose levels were in fact within healthy ranges for healthy participants. For the participant with diabetes, glucose was found to be above the healthy range while the protein level was in the healthy range.
\end{abstract}

$\mathrm{D}$ iseases surround the world and being able to diagnose them is crucial in order to then cure the disease. Diagnosing diseases is not always an affordable, time-efficient, or easy task. Paper-based microfluidic devices construct a framework that leaves the level of complication and cost low while keeping the efficiency high. ${ }^{1-6}$ The application of paperbased microfluidics presents many advantages such as their cost effectiveness: they are easy to mass-produce, transport, store, implement, dispose, and they do not need excessive equipment to move liquid like other devices. There are also many disadvantages to paper-based microfluidic devices. They are relatively new, which means issues such as the control over flow rates, mixing, and interaction times between sample and reagents have not yet been perfected. ${ }^{7-9}$

A previous approach by Liu and Crooks ${ }^{10}$ uses photolithography and origami to develop a 3D paper-based analytical device $(\mu \mathrm{PAD})$. The inclusion of origami to the overall design of the $\mu \mathrm{PAD}$ allows for only one step of the fabrication process. The $\mu \mathrm{PAD}$ can be simply folded into multiple layers. This also eliminates the contamination and nonspecific adsorption caused by using tape in previous $3 \mathrm{D}$ designs. ${ }^{3}$ Following the procedure, analysis can be done by unfolding the $\mu \mathrm{PAD}$, revealing the colorimetric and fluorescence assays on each layer. Photolithography patterns the paper by use of a light-sensitive chemical as photoresist, creating a pattern on the paper to control fluid flow without any input of excess energy such as a pump or valve system in glass-based microfluidic systems to direct or manipulate the fluid. Still using only small samples from the test subject, approximately $5 \mu \mathrm{L}$, allows for multiple diagnostic tests on one $\mu \mathrm{PAD}$, resulting in an inexpensive, portable device. $^{11}$

Like photolithography, wax printing is a simple and inexpensive method of fabricating a $\mu \mathrm{PAD}$, as discussed by Whitesides and co-workers. ${ }^{3}$ Fabrication by wax printing involves only two main procedures: the first step is printing patterns of wax on the surface of paper, and the second is melting the wax into the paper to form hydrophobic barriers. This can prevent any mixing of the fluids passing through the device. The low cost of production and low levels of complexity to create these paper-based analytical devices make them wellsuited to be manufactured at a large scale for prototyping. The features are not as accurate as other methods such as photolithography but are sufficient for uses such as detecting substances due to color change in the test assay. The melting of the wax is both lateral and vertical, spreading across the paper and creating a hydrophobic barrier across the thickness of the paper.

Most published work focuses on ready-to-use microfluidic devices. For instance, paper-based blood typing devices can be used to detect the presence or absence of hemagglutination reactions, which can lead to rapid identification of human blood type at a significantly lower cost than current mainstream

Received: May 16, 2013

Accepted: October 23, 2013

Published: October 23, 2013 
(a)

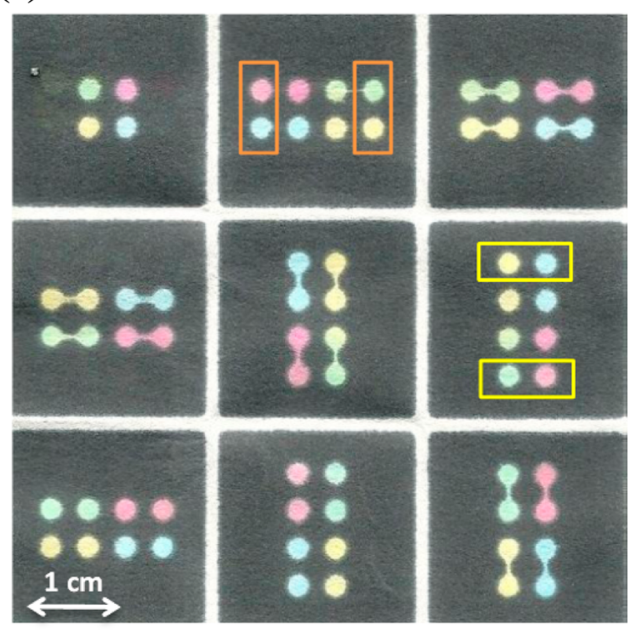

(b)

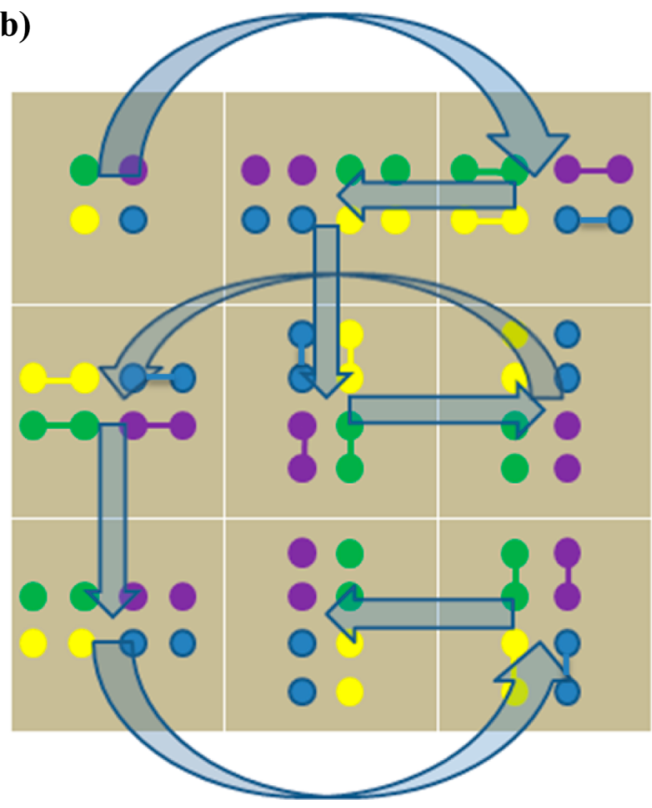

Figure 1. (a) Unfolded paper-based microfluidic device made by wax printing technique. Detection reservoirs are shown by yellow rectangles for protein and orange rectangles for glucose. The image shows the flow of colored solutions from the entrance points to the bottom layers. (b) Flow pattern of the microfluidic device. Sample is introduced in the top left corner and ends in the bottom middle square.

methods. These tests can be operated without the assistance of medical personnel, making these devices attractive for diagnostic applications in remote locations and the developing world. $^{12}$ Other applications for these devices include simultaneous detection of analytes within a test sample and microfluidic origami chemiluminescence detection system for point-of-care (POC) diagnostics. ${ }^{13}$ There are multiple techniques to create these devices including photolithography plotting, ${ }_{16}^{11,14}$ plasma oxidation, ${ }^{14}$ flexography printing, ${ }^{14}$ wax printing, ${ }^{16}$ and inkjet printing. ${ }^{4,15}$ Along with the number of techniques utilized, there is a wide variety of hydrophobic substances available for creating channels on hydrophilic paper, each with its own advantages and disadvantages.

Wax printing and ink jet printing are proving to be the most promising of fabrication methods due to their low-cost patterning and quick fabrication process. Wax printing is also an ideal form of production for mass prototyping, which is essential for low-cost portable diagnostic devices that could be used in remote locations in developing regions. Ideally, we are interested in diagnostic devices that do not require extra equipment or power to operate. Overall, the advantages of paper-based diagnostic devices may become a driving force for rapid, cheap, flexible, and reliable devices to be developed for clinical emergency as well as large-scale use in developing regions where reliability, affordability, and user friendliness are major challenges.

This paper focuses on the use of wax printing and basic origami folding technique to fabricate $3 \mathrm{D} \mu \mathrm{PADs}$ and its advantages over previous fabrication methods because of its low -cost, simplicity, and capability to produce large amounts of prototypes rapidly. The nine-layer platform along with the basic yet versatile reservoir and channel design allows for several analyte to assay combinations that can be easily tailored to a specific region of the world, or a certain physician's expertise, where a certain set of diagnoses are specifically sought after. These changes can be made with minimal effort and effect on the reproducibility of the device's performance. ${ }^{10}$ The design can be set to simultaneously test urine, blood, and saliva samples in their respective assays, or a single analyte can be tested to its full extent. For example, urine can be colorimetrically tested for glucose, protein, nitrites, bilirubin, urobilinogen, ketones, erythrocytes, and leukocytes. ${ }^{17}$

We specifically use the prototype developed by wax printing to test protein and glucose levels within a human urine sample. The design of the prototype $\mu \mathrm{PAD}$ requires very small sample sizes; in the case of a urine sample, $3 \mu \mathrm{L}$ is all that is necessary.

The $\mu \mathrm{PAD}$ proved to be cost-effective in its fabrication, to maintain reliability when mass-produced, to have a simple procedure for its administration, and to have the ability to give several diagnoses per limited amount of test sample. This device could also be integrated with technologies such as lensless imaging ${ }^{18,19}$ for POC diagnostics in resource-limited regions. For users who do not have access to scanners and programs to manipulate the results, the device still can be used to qualitatively evaluate the color change. For instance, a simple colorimetric guide can accompany the device depicting sequences of sequential color change respectively matched alongside the corresponding compound concentration range which gives each description, quantitatively predetermined in the lab. This way, the user can simply match color schemes from the guide to the test sample.

\section{MATERIALS AND METHODS}

In order to make this device cost-efficient while still being portable, minimal materials are necessary. The first step to create the microfluidic device is to choose the paper on which they are printed. The paper needs to allow the mixtures to flow but also have barriers that can control the flow. As discussed by Whitesides and co-workers, ${ }^{20}$ wax printing is very affordable and easy to use by utilizing a standard printer (Xerox ColorQube 8570DN) that prints wax instead of ink. Previous studies chose Whatman no. 1 chromotography paper (costing about $\$ 7 / \mathrm{m}^{2}$ and available in sheets of $460 \mathrm{~mm} \times 570 \mathrm{~mm}$ ) due 
to its hydrophilic and biocompatible behavior as well as low cost.

The design was first created in SolidWorks 2011 and then printed onto the paper. Once the wax was printed onto the paper, it was put into an oven at $150{ }^{\circ} \mathrm{C}$ for $120 \mathrm{~s}$. This heating process allows the wax to melt into the Whatman no. 1 chromotography paper both vertically and horizontally, creating a hydrophobic blockade throughout the paper forming a barrier. This works for both the lateral flow devices and 3D models. After the paper was chosen, the thickness of the ink line was tested for the required width of line to create a fully hydrophobic barrier. This was tested by drawing five rectangles and performing the fabrication process with different beginning line widths. Colored water was placed in the center of the rectangles after the wax was melted. It was found that the smallest original line width, while still maintaining a hydrophobic barrier, was $400 \mu \mathrm{m}$, and after melting it was expanded to $1000 \mu \mathrm{m}$. The simplicity of the device allows the overall cost to be minimal.

The next step was preparing the assays for the detection points. Two assay kits were purchased from Sigma-Aldrich. The assays purchased were (TP0100) for protein and (GAGO20) for glucose. With the glucose assay being used as is, the protein assay needed to be prepared by dilution. The ratio used was 4:1 distilled water to protein.

By use of the $3 \mathrm{D}$ origami technique, a prototype can be created by folding the device into a three-dimensional shape. Because of the hydrophobic channels created from the wax printing technique, the sample flows through the channels toward the detection points. The $3 \mathrm{D}$ model is important because it flows in the $x, y$, and $z$ directions, allowing multiple detection points on a small footprint. The glucose and protein assays were placed on different layers of the device (outlined with rectangles as shown in Figure 1a), prior to the experiment and were allowed to dry at $20{ }^{\circ} \mathrm{C}$ for $30 \mathrm{~min}$. Once the assays were dry, the device was placed in four plastic corner sleeves to ensure tight contact between layers, and a sample $(3 \mu \mathrm{L})$ of urine was introduced at the entrance point to the nine-layer device, with fluid flow directed in the three dimensions. To gauge the effect of protein and glucose levels on the color change in the assays, synthetic urine with predetermined levels was analyzed along with actual urine samples from four volunteers. Synthetic urine samples with protein and glucose were prepared separately and tested simultaneously on one $\mu \mathrm{PAD}$.

Subsequently, individual human urine samples were simultaneously tested for protein and glucose levels on the same $\mu \mathrm{PAD}$. The samples were allowed to completely react until dry with the glucose and protein assay to ensure the best beforeand-after evaluation of color change. Once the reaction was complete, the device was unfolded to observe the testing assays for colorimetric analysis. Scans were taken immediately after drying due to the color fading in the detection points after 20 $\mathrm{min}$. The color in the detection points after reaction occurred was captured by use of a HP Scanjet $6300 \mathrm{c}$ with a resolution of $600 \mathrm{dpi}$. Multiple devices can be scanned at once into the same file. Once scanned, the pictures were uploaded into Photoshop to measure the color change that occurred from mixing. When mixed with urine, the glucose assay changes from clear to brown, while the protein assay changes from brown to a lighter shade of brown. The changing color of glucose is considered the same color with only the shade changing; this was analyzed with grayscale. Color was discarded in Photoshop by selecting grayscale. The histogram tool was used to show the brightness or darkness level of a certain area. The part of the picture that was being analyzed was then selected, including only the colored parts of the detection zone and omitting the ink outline. By clicking in the middle of the selected box and dragging it, the same amount of pixels can be used for every branch. The mean average brightness with the amount of pixels will then be shown on the histogram box. From this, the color change was quantified for the results. For glucose, the grayscale shade change was recorded, and for protein, the RGB (red, green, blue) averages were recorded.

\section{RESULTS AND DISCUSSION}

In order to test the device, first we studied the correlation of color change and the known amount of protein and glucose in synthetic urine. A graph was created by plotting the exact color change in pixels versus the amount of glucose and protein in the synthetic urine sample (Figures 2 and 3 ). A best-fit line was

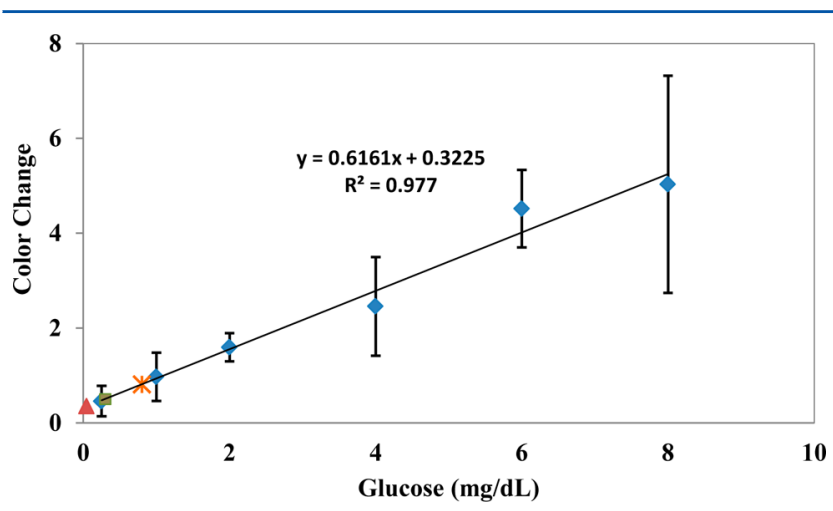

Figure 2. Color change as a function of known amounts of glucose (blue diamonds). This creates a scale that can be referenced when experiments are conducted. Based on the color change measured in Photoshop, participant 1 (green square) displayed a color change of 0.5 pixel, equivalent to $0.3 \mathrm{mg} / \mathrm{dL}$ glucose. Participant 2 (red triangle) displayed a color change of 0.35 pixel, indicating a value of $0.05 \mathrm{mg} / \mathrm{dL}$ glucose. Participant 3 (orange asterisk), displayed a color change of 0.81 pixel or $0.8 \mathrm{mg} / \mathrm{dL}$ glucose. Participant 4 (not shown) displayed a color change of 71 pixels or $114.8 \mathrm{mg} / \mathrm{dL}$ glucose. Normal glucose range in urine must be below $15 \mathrm{mg} / \mathrm{dL}$.

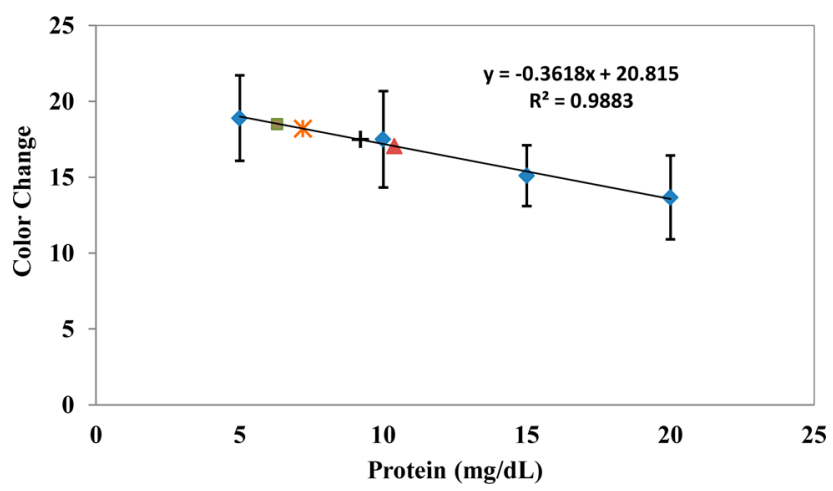

Figure 3. Relationship between color change and amount of protein in urine samples (blue diamonds). Protein levels of participants 1-4 are shown as green square, red triangle, orange asterisk, and black plus sign, respectivelyf. The average color change ranges from 18.5 to 17 pixels, indicating protein levels that range from 6.3 to $10.4 \mathrm{mg} / \mathrm{dL}$. Normal protein range in urine is less than $10 \mathrm{mg} / \mathrm{dL}$. 
Table 1. Protein and Glucose Levels Measured by 3D Paper Device, Laboratory Urinalysis Testing, and Commercial Glucose Meter

\begin{tabular}{|c|c|c|c|c|c|c|c|}
\hline \multirow[b]{2}{*}{ participant } & \multicolumn{4}{|c|}{ glucose } & \multicolumn{3}{|c|}{ protein } \\
\hline & $\begin{array}{l}\text { color change } \\
\text { (pixels) }\end{array}$ & $\begin{array}{c}\text { in urine }(\mathrm{mg} / \\
\mathrm{dL})\end{array}$ & $\begin{array}{l}\text { lab urinalysis } \\
\text { testing }^{a}\end{array}$ & $\begin{array}{c}\text { in } \text { blood }^{b}(\mathrm{mg} / \\
\mathrm{dL})\end{array}$ & $\begin{array}{l}\text { color change } \\
\text { (pixels) }\end{array}$ & $\begin{array}{c}\text { in urine }(\mathrm{mg} / \\
\mathrm{dL})\end{array}$ & $\begin{array}{l}\text { lab urinalysis } \\
\text { testing }^{a}\end{array}$ \\
\hline 1 & 0.5 & 0.3 & neg & 74 & 18.5 & 6.3 & neg \\
\hline 2 & 0.35 & 0.05 & neg & 72 & 17 & 10.4 & neg \\
\hline 3 & 0.81 & 0.8 & neg & 82 & 18.2 & 7.2 & neg \\
\hline 4 & 71 & 114.8 & trace & 143 & 17.5 & 9.2 & neg \\
\hline
\end{tabular}

${ }^{a}$ Negative (neg) represents normal urine containing little or no protein or glucose. Trace represents glucose level of $100 \mathrm{mg} / \mathrm{dL}$ in urine sample. ${ }^{b} \mathrm{By}$ ACCU-CHEK Compact Plus.

applied to the data, giving an equation for the line. This can be used later to determine the amount of protein or glucose each participant had in their urine sample. A wide spectrum of results was obtained by testing different amounts of glucose and protein in synthetic urine and the effect each one had on color change. For glucose the amounts chosen were $0.25,1,2,4,6$, and $8 \mathrm{mg} / \mathrm{dL}$. The healthy range for glucose is less than $15 \mathrm{mg} /$ $\mathrm{dL}$, so the amounts we chose were to establish the trend within that range.

For protein, standard amounts of 5, 10, 15, and $20 \mathrm{mg} / \mathrm{dL}$ were used. The healthy range for protein levels is less than 10 $\mathrm{mg} / \mathrm{dL}$. There are a number of factors that could lead to higher levels of protein, as opposed to glucose, where the main factor would be diabetes. For this reason we chose protein levels in the synthetic samples outside the health zone to accommodate for the seemingly healthy individual. Each experiment was performed five times to find an average and standard deviation for sets of data.

Once the correlation between color change and the amount of protein or glucose was found, actual urine samples from four volunteers were analyzed. Of these volunteers, two were male and two were female, ages $21-48$ with a weight range of $120-$ 215 lbs. The samples were taken before breakfast. Three of the participants had no pre-existing health conditions documented. Each participant's sample was tested by the three-dimensional device by the same method as the synthetic urine. A commercial blood glucose meter, ACCU-CHEK Compact Plus, was employed as another validation technique. The samples were also sent to a laboratory for urinalysis testing. Reagent strips were used for testing. Each strip tests for two markers, glucose and protein. The amount of glucose and protein in the urine and blood samples of each volunteer was measured by all three techniques.

The results show that the participants with no pre-existing health conditions were in the healthy ranges of protein and glucose levels. The glucose level in urine for the participant with diabetes (control) was found to be $114.8 \mathrm{mg} / \mathrm{dL}$, which is not shown in Figure 2. The protein level for this person was found to be $9.2 \mathrm{mg} / \mathrm{dL}$ and is shown as a black plus sign in Figure 3.

The danger zone or unhealthy zone for protein is more than $10 \mathrm{mg} / \mathrm{dL}$ protein in the urine. The presence of a larger amount of protein in urine may be related to kidney diseases such as glomerulonephritis. It may also be due to illnesses such as heart failure, high blood pressure, or bladder tumor. Having glucose in the urine can be a sign of diabetes. The appropriate level of glucose that is allowed in urine sample is in the range $0-15 \mathrm{mg} / \mathrm{dL}$.

Table 1 summarizes the amounts of protein and glucose measured by our 3D device, laboratory urinalysis testing, and a commercial glucose meter. In laboratory tests, "neg” represents normal urine containing little or no protein or glucose and "trace" represents a glucose level of $100 \mathrm{mg} / \mathrm{dL}$ in urine sample. Both laboratory tests and our 3D device showed that the glucose levels were pretty small for participants $1-3$ and about $100 \mathrm{mg} / \mathrm{dL}$ for participant 4. Fasting blood sugar above 130 $\mathrm{mg} / \mathrm{dL}$ is reported as diabetes by the American Diabetes Association (ADA). The glucose levels in urine samples were very low compared to the blood samples. However, the results follow the same trend. Participants 1-3 were found to have glucose amounts below $1 \mathrm{mg} / \mathrm{dL}$ in their urine samples and their blood glucose levels were also in the healthy range. The glucose level for participant 4 was above the indicated healthy range in both blood and urine samples. The protein level for all participants was in the healthy range as measured by both our 3D paper device and laboratory urinalysis testing.

From the results in this experiment, it can be seen that a logical estimation can be made of protein and glucose levels with a simple diagnostic test using a paper microfluidic device. By experimenting with known amounts of glucose and protein initially, a relationship was determined between the average color change and the actual quantities in the sample. A quick and easy test of a urine sample and referencing the relationship on the graph will give an estimation of protein and glucose amounts. Although only a few layers were needed for this simple test of urine samples, it is apparent that more complex studies and tests can be performed by using the inexpensive fabrication method of wax printing and applying the origami technique. To be able to analyze more substances within the fluid, more layers within the device would be needed. Since all layers are fabricated at the same time, the construction of more will not pose much of an obstacle.

\section{CONCLUSIONS}

It is shown that paper-based microfluidic devices have lots of potential. They are flexible, lightweight, biodegradable, and can be extraordinarily cheap. Paper can reach what seems like an endless range of properties whether it is hydrophobic, hydrophilic, porous, watertight, , or transparent. In terms of microfluidics, the versatility of paper leaves a lot of room for creativity in the design of 3D assays. This paper focused on creating a paper-based microfluidic device that has a high degree of utility, is user-friendly, efficient to produce, and even easy to customize. With the use of origami and wax printing, a microfluidic device was created that required very little test sample, simple administration, and little time to use. To demonstrate the effectiveness of the device, assays were prepared to test human urine samples and to give an estimation of glucose and protein amounts in the samples. Synthetic urine samples with predetermined levels of protein and glucose were 
also prepared to calibrate the effects on the color change. Urine samples from four participants with and without pre-existing health conditions were collected and analyzed. The results affirmed that both protein and glucose amounts were in healthy ranges for participants with no health conditions. The glucose level for the participant with diabetes was found to be above the healthy range while the protein level was in the healthy range. Overall the effectiveness, simplicity, and versatility of the origami paper-based microfluidic device is shown to be a viable tool with great potential in POC diagnostics.

\section{AUTHOR INFORMATION}

\section{Corresponding Author}

*E-mail nastaran@iastate.edu.

\section{Notes}

The authors declare no competing financial interest.

\section{ACKNOWLEDGMENTS}

We thank Marie Kuhlmann and Luke Wagner for their assistance with this project. This work was supported by the William March Scholar program and the Iowa State University Foundation.

\section{REFERENCES}

(1) Osborn, J. L.; Lutz, B.; Fu, E.; Kauffman, P.; Stevens, D. Y.; Yager, P. Lab Chip 2010, 10, 2659.

(2) Siegel, A. C.; Phillips, S. T.; Dickey, M. D.; Lu, N.; Suo, Z.; Whitesides, G. M. Adv. Funct. Mater. 2010, 20, 28.

(3) Martinez, A. W.; Phillips, S. T.; Whitesides, G. M. Proc. Natl. Acad. Sci. 2008, 105, 19606.

(4) Abe, K.; Suzuki, K.; Citterio, D. Anal. Chem. 2008, 80, 6928.

(5) Ballerini, D. R.; Li, X.; Shen, W. Microfluid. Nanofluid. 2012, 13, 769.

(6) Wagner, L. T.; Hashemi, N.; Hashemi, N. ESFuelCell2013-18322, Minneapolis, MN, 2013.

(7) Hashemi, N.; Erickson, J. S.; Golden, J. P.; Ligler, F. S. Biomicrofluidics 2011, 5, No. 032009.

(8) Hashemi, N.; Erickson, J. S.; Golden, J. P.; Jackson, K. M.; Ligler,

F. S. Biosens. Bioelectron. 2011, 26, 4263.

(9) Noh, H.; Phillips, S. T. Anal. Chem. 2010, 82, 4181.

(10) Liu, H.; Crooks, R. M. J. Am. Chem. Soc. 2011, 133, 17564.

(11) Martinez, A. W.; Phillips, S. T.; Butte, M. J.; Whitesides, G. M. Angew. Chem., Int. Ed. 2007, 46, 1318.

(12) Jarujamrus, P.; Tian, J.; Li, X.; Siripinyanond, A.; Shiowatana, J.; Shen, W. Analyst 2012, 137, 2205.

(13) Ge, L.; Wang, S.; Song, X.; Ge, S.; Yu, J. Lab Chip 2012, 12, 3150.

(14) Li, X.; Ballerini, D. R.; Shen, W. Biomicrofluidics 2012, 6, 011301.

(15) Abe, K.; Kotera, K.; Suzuki, K.; Citterio, D. Anal. Bioanal. Chem. 2010, 398, 885.

(16) Delaney, J. L.; Hogan, C. F.; Tian, J.; Shen, W. Anal. Chem. 2011, 83, 1300.

(17) Lee, D.-S.; Jeon, B. G.; Ihm, C.; Park, J.-K.; Jung, M. Y. Lab Chip 2011, 11, 120 .

(18) Moon, S.; Keles, H. O.; Ozcan, A.; Khademhosseini, A.; Hæggstrom, E.; Kuritzkes, D.; Demirci, U. Biosens. Bioelectron. 2009, 24, 3208.

(19) Ozcan, A.; Demirci, U. Lab Chip 2008, 8, 98.

(20) Carrilho, E.; Martinez, A. W.; Whitesides, G. M. Anal. Chem. 2009, 81, 7091 . 